\title{
The Swift Mission
}

\author{
Alberto Moretti ${ }^{1,2}$ \\ ${ }^{1}$ INAF-Osservatorio Astronomico di Brera, via E. Bianchi, 23807 Merate(LC), Italy \\ ${ }^{2}$ On behalf of the Swift Team
}

\begin{abstract}
The Swift Gamma-ray Burst Explorer mission, launched on 2004 November 20, is a multiwavelength observatory for gamma-ray burst (GRB) astronomy. The satellite carries three instruments: a new-generation wide-field gamma-ray (15-150 keV) detector that detects bursts, calculates 1-4 arcmin positions, and triggers autonomous spacecraft slews; a narrow-field X-ray telescope that gives 5 arcsec positions and performs spectroscopy in the 0.2 to $10 \mathrm{keV}$ band; and a narrow-field UV/optical telescope that operates in the 170-600 $\mathrm{nm}$ band and provides 0.3 arcsec positions and optical finding charts. In the first 8 months of the mission (until the end of July 2005), Swift detected 54 GRBs performing detailed X-ray and UV/optical afterglow observations spanning timescales from 1 minute to several days after the burst. Swift has already collected a rich trove of early X-ray afterglow data and some interesting features are emerging. In particular early afterglow signatures reveal valuable and unprecedented information about GRBs, including the prompt emission - afterglow transition, GRB emission site, central engine activity, forward-reverse shock physics, and the GRB immediate environment.
\end{abstract}

Keywords. Swift satellite, Gamma Ray Burst.

\section{Introduction}

Gamma-ray bursts (GRBs) were discovered in the late 1960s in data from the Vela satellites. For over 25 years following their discovery GRBs remained one of the greatest mysteries in astrophysics, largely due to a frustrating inability to pinpoint their locations on the sky and to detect any counterparts at longer wavelengths. This situation changed dramatically in February 1997, when the Beppo-SAX satellite discovered the first X-ray afterglow of a GRB, confirming the teoretical predictions that X-ray, optical, and radio afterglows are produced by the expanding relativistic fireball that produces the burst itself. For long bursts, the discovery by BeppoSAX and ground-based observers of X-ray through radio afterglow allowed redshifts to be measured and host galaxies to be found, proving a cosmological origin. GRB correspond to a huge instantaneous energy release of $10^{51}-10^{52}$ ergs and are probably related to black hole formation, possibly related to endpoints of stellar evolution; they also represent bright beacons from the high redshift universe. By the end of 2004 Beppo-SAX, RXTE, ASCA, Chandra, and XMM-Newton had found $55 \mathrm{X}$-ray afterglows. The mission planning requirements of these satellites have typically led to delays of 6-24 hours between the burst and the afterglow detection, during which time the afterglow emission fades by many orders of magnitude. Swift (Gehrels et al. 2004) was designed to fill in the gap by making very early multiple wavebands observations of afterglows, beginning approximately a minute after the discovery of a GRB. Since afterglows fade quickly, typically as $t^{-1}$ or $t^{-2}$, Swift's rapid $\sim 1$ minute response allows observations when the emissions are orders of magnitude brighter than the previous few-hour response capabilities. 


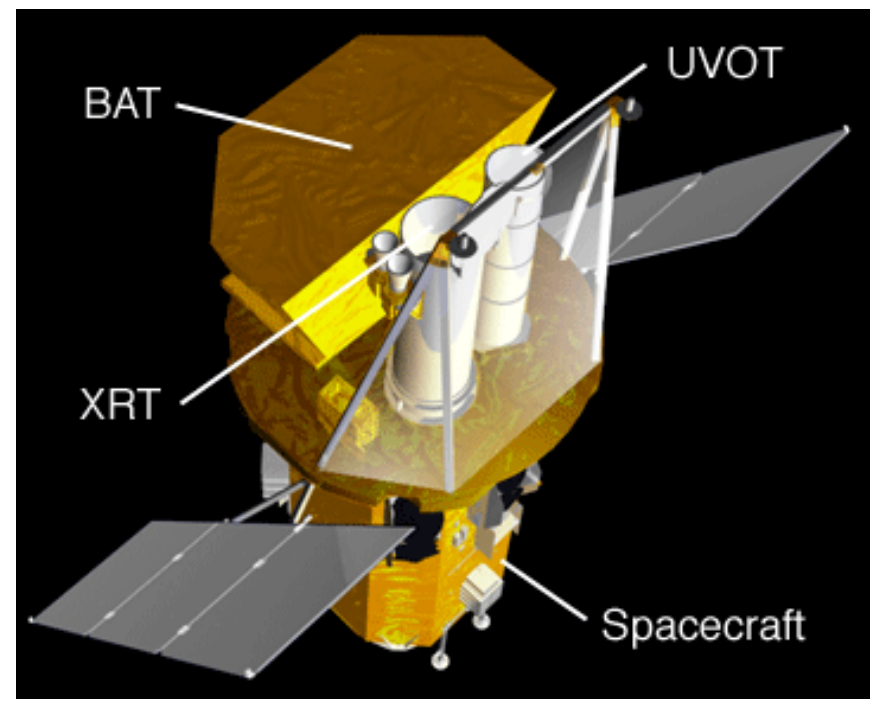

Figure 1. The Swift satellite.

\section{Swift Mission}

Swift's science payload consists of three instruments mounted onto an optical bench. These instruments, the Burst Alert Telescope (BAT), X-Ray Telescope (XRT) and UltraViolet/Optical Telescope (UVOT), are shown on the spacecraft in Figure 1. The launch was on a Delta $7320-10$ on 2004 November 20 to a 22 degrees inclination, 600-km altitude orbit. Swift has a nominal lifetime of 2 years with a goal of 5 years and an orbital lifetime of $\sim 8$ years. Normal data are downlinked in several passes each day over the Italian Space Agency (ASI) ground station at Malindi, Kenya. Tracking and Data Relay Satellite System (TDRSS) are used to send burst alert messages to the ground. Similarly, information about bursts observed by other spacecraft are uplinked through TDRSS for evaluation by Swift's on-board figure-of-merit (FoM) software. There is no on-board propulsion system. Pointing is provided by momentum wheels with momentum unloaded by magnetic torquers. Pointing knowledge is through the gyroscopes, star trackers, sun sensors, and magnetometers. The observation strategy is for the XRT and UVOT to be almost constantly observing positions of bursts previously detected by the BAT. The most recently detected burst have priority (although this can be adjusted as science dictates). Typically 5 sources are observed each orbit. When a burst is detected, an automated series of XRT and UVOT observations are performed lasting 20,000 s in exposure. Following that time, additional observations are scheduled via ground planning.

\subsection{Burst Alert Telescope}

The Burst Alert Telescope (BAT) is a highly sensitive, large FOV instrument designed to provide critical GRB triggers and 4-arcmin positions. It is a coded-mask instrument with a 1.4 steradian field-of-view (half coded). The energy range is $15-150 \mathrm{keV}$ for imaging with a non-coded response up to $500 \mathrm{keV}$. Within the first $\sim 10$ seconds of detecting a burst, the BAT calculates an initial position, decides whether the burst merits a spacecraft slew and, if worthy, sends the position to the spacecraft. Further information on the XRT is given by Barthelmy et al. (2004). Since the BAT coded FOV always includes the XRT and UVOT fields-of-view, long duration gamma-ray emission from the burst can be studied simultaneously with the X-ray and UV/optical emission. When a burst is detected, the 


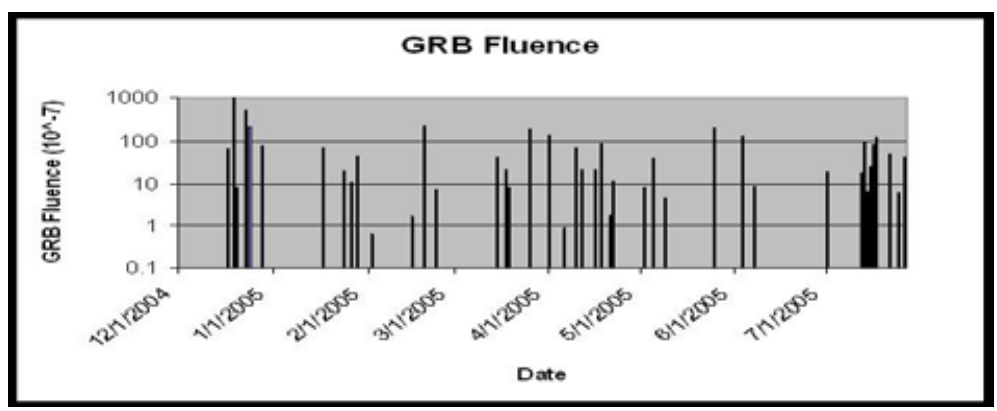

Figure 2. The temporal distribution of the GRB detected by Swift, with an indication of the flux measurements.

sky location and intensity are immediately sent to the ground and distributed to the community through the Gamma-Ray Burst Coordinates Network (GCN). The data from the BAT are also producing a sensitive hard X-ray all-sky survey over the course of Swift's two year mission.

\subsection{X-Ray Telescope}

Swift's X-Ray Telescope (XRT) is a focusing X-ray telescope with a $110 \mathrm{~cm}^{2}$ effective area (at $1.5 \mathrm{keV}$ ), 23 arcmin FOV, 18 arcsec resolution (half-power diameter at $1.5 \mathrm{keV}$ ), and $0.2-10 \mathrm{keV}$ energy range. XRT is designed to measure the fluxes, spectra, and lightcurves of GRBs and afterglows over a wide dynamic range covering more than 7 orders of magnitude in flux. The XRT pinpoints GRBs to 5-arcsec accuracy within 10 seconds of target acquisition for a typical GRB and studies the X-ray counterparts of GRBs beginning 50-200 seconds from burst discovery and continuing for days to weeks. Further information on the XRT is given by Burrows et al. (2004).

\subsection{Ultra-Violet/Optical Telescope}

The Ultra-Violet/Optical Telescope (UVOT) design is based on the Optical Monitor (OM) on-board ESA's XMM-Newton mission. UVOT is co-aligned with the XRT and carries an 11-position filter wheel, which allows low-resolution grism spectra of bright GRBs, and broadband UV/visible photometry. There is also a $4 \times$ field expander (magnifier) that delivers diffraction limited sampling of the central portion of the telescope FOV. Photons register on the microchannel plate intensified CCD (MIC). Further information on the UVOT is given by Roming et al. (2004).

\section{Statistics in the first 8 months of the mission}

Swift was successfully launched on 2004 November 20. The BAT first light was on the 3rd of December 2004, XRT first light was on the 11th of December 2004 and UVOT first light was on the 12th of January for UVOT. The first GRB was detected by BAT on the 17th of December 2004, while the first X-ray afterglow was detected by XRT on the 23rd of the same month. The first optical afterglow was detected by UVOT on the 15th of March 2005. The verification and calibration phase has ended on the 5th of April 2005 and since then all the data from the 3 instruments are publicly available together with the reduction and analysis software (http://swift.gsfc.nasa.gov/sdc/). In the first 8 months of the mission (until the end of July 2005), BAT detected 54 bursts (Fig. 2). Among them 3 were classified as short bursts, 7 as X-ray flash (XRF). The extrapolated detection number per year is $94 \pm 10$. XRT detected 40 X-ray afterglows 

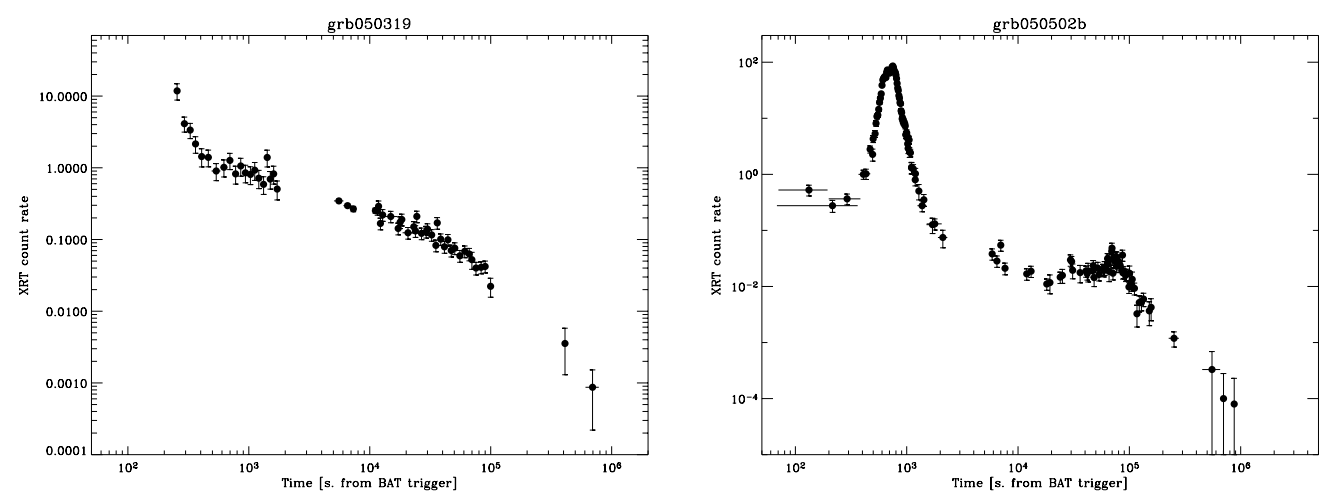

Figure 3. The lightcurve of the afterglows of the GRB 050319 (left, Cusumano et al. 2005) and 050502b (right, Falcone et al. 2005).

out of 41 pointings: 25 of them were observed within 300 seconds from the BAT trigger. In the same period UVOT detected 8 afterglows out of 37 pointings. For 21 bursts an optical counterpart was found by UVOT and/or optical ground telescopes. The average distance between the X-ray positions calculated from XRT data and the 21 afterglows with optical counterparts is 3.5 arcseconds. The typical distance between the BAT and XRT position is 100", much better than the expectations. For 12 bursts of this sample was possible to measure the redshift: the average redshift of this subsample is $\sim 2$ (the average redshift of Beppo-SAX 55 bursts sample was $~ 1)$ with 4.3 as maximum measured redshift.

\section{Preliminary striking results: the X-ray afterglow light curves}

Before Swift, X-ray afterglow emission was detected, in most cases, only several hours after the burst, by which time the flux typically showed a smooth single power law decay $\sim t^{-1}$. However, the early afterglow evolution - the first few hours, which can probe important questions such as the density profile of the external medium and the early radiative energy losses from the external shock, remained largely unexplored. From the observation of the first months of the Swift mission, a general trend starts to emerge that may become the standard to describe each GRB X-ray afterglow light curve (Chincarini et al. 2005, Nousek et al. 2005). Starting at the earliest XRT observations (approximately $10^{2} \mathrm{~s}$ after the prompt gamma-rays), the X-ray flux $F_{\nu}$ follows a canonical behavior comprising three power law segments where $F_{\nu} \propto \nu^{-\beta} t^{-\alpha}$ (see Fig. 3): an initial steep decay slope $\left(\alpha_{1}\right)$ (Tagliaferri et al. 2005, Perri et al. 2005), which (at $t_{\mathrm{break}, 1}$ ) changes into a very flat decay $\left(\alpha_{2}\right)$, that in turn (at $\left.t_{\mathrm{break}, 2}\right)$ transitions to a slightly steeper slope $\left(\alpha_{3}\right)$. The spectrum remained constant throughout the breaks (within our available statistics) in all cases except two (GRBs 050315 and 050319) where the spectrum hardened (i.e. $\beta$ decreased) across the first break (at $t_{\text {break,1 }}$ ). It should be noted that the values of $\alpha_{3}$ are consistent with those seen in previous missions since they typically started observations hours after the burst (Campana et al. 2005, Moretti et al. 2005).

The most promising explanation for the initial fast flux decay $\left(\alpha_{1}\right)$ is that it is the tail of the prompt gamma-ray emission which is emitted from large angles $\left(\theta>\Gamma^{-1}\right)$ relative to our line of sight. This model produces a sharp flux decay with $\alpha_{1}=2+\beta_{1}$, in rough agreement with observations, while $\alpha_{1}>2+\beta_{1}$ might also be expected for $t / T_{\mathrm{GRB}} \lesssim \mathrm{a}$ few. The shallow intermediate flux decay $\left(\alpha_{2}\right)$ is most likely caused by 


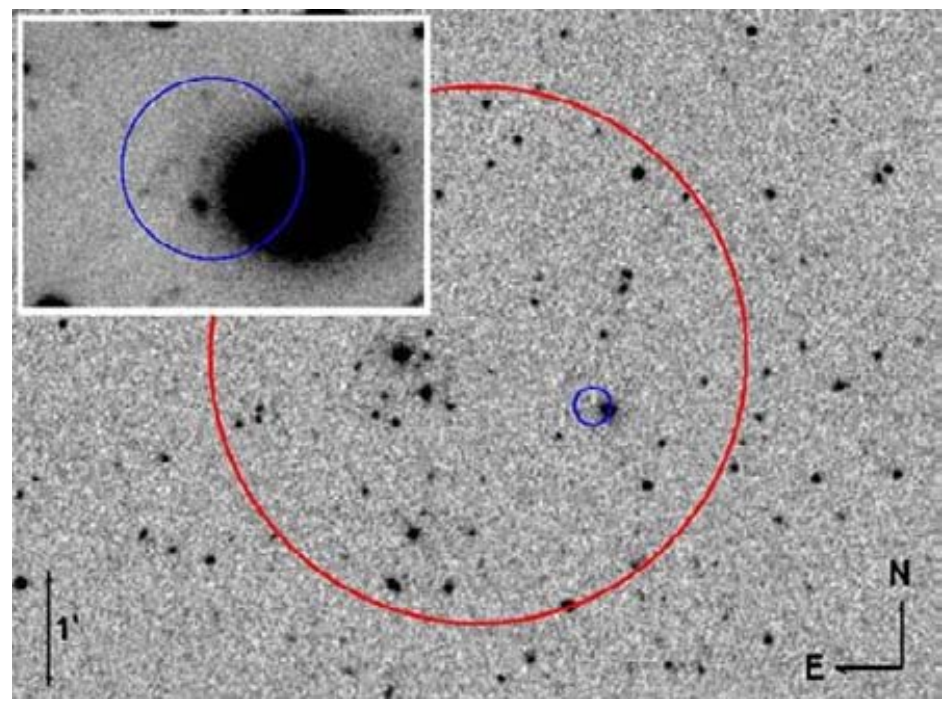

Figure 4. The XRT (small circle) and BAT (large circle) error circles of the short GRB $050509 \mathrm{~b}$ overplotted on the optical image.

continuous energy injection into the forward shock. This energy injection is probably due to a decrease in the Lorentz factor, $\Gamma$, of the outflow toward the end of the prompt GRB, resulting in a monotonic increase of $\Gamma$ with radius. This outflow gradually catches up with the afterglow shock, resulting in a smooth energy injection. The third power law segment of the light curve $\left(\alpha_{3}\right)$ is most likely the well known afterglow emission from a spherical adiabatic external shock. The observed values of the temporal index $\left(\alpha_{3}\right)$ and the spectral index $\left(\beta_{3}\right)$ are consistent with this interpretation.

The early X-ray light curves obtained with Swift XRT often show flares (Burrows et al. 2005, Romano et al. 2005). The most prominent flare so far was in GRB 050502b (Fig. 3), where the flux increased by a factor of $\sim 500$. Some of these flares have very sharp temporal features where the flux changes significantly on time scales $\Delta t \ll t$. Most flares have a very steep rise and decay (with very large temporal rise/decay indices when fitted to a power law). When the flare is bright enough to follow its spectral evolution, its hardness ratio evolves during the flare, and its spectral index is somewhat different than the one associated with the underlying power law decay of the X-ray light curve before and after the flare. Furthermore, the fluxes before and after the flare lie approximately on the same power law decay, suggesting that the flare originates from a distinct physical component than that responsible for the underlying power law decay. These flares are most likely caused by internal shocks within the outflow that is ejected from the central source at late times (very close to the time when these flares are seen). This implies the the central source quite often remains active for hours after the GRB.

\section{The origin of the short GRBs}

The duration of GRBs has long been known to show a bimodal distribution; moreover short bursts are predominantly harder than the longer variety. Most of the progress to date on understanding the origin of GRBs has been for long bursts. Before Swift, no short GRB had been accurately and rapidly located, although arcminute-sized error boxes had been searched unsuccessfully hours to days after the event. Theoretical predictions for the cause of short GRBs have included neutron star - neutron star (NS-NS) or NS - black hole 
(BH) mergers, extragalactic soft gamma repeater giant flares, or collapsars/hypernovae with different explosion or beaming properties than those responsible for the long bursts.

Swift has provided the first accurate localization of a short GRB, the GRB 050509b (Gehrels et al. 2005). No optical afterglow was detected and Chandra did not detect the X-ray afterglow 2 days later. The center of the XRT error circle lies only 9.8 " away from the center of the large E1 elliptical galaxy 2MASX J12361286+2858580 at a redshift of $\mathrm{z}=0.225$, which is located in the galaxy cluster NSC J123610+285901 (Fig. 4). The association with this galaxy seems unlikely to be coincidental. Infact, galaxies as bright as this are relatively rare; the comoving number density of galaxies at least this luminous is $5 \times 10^{-5} \mathrm{Mpc}^{-3}$; the probability of lying within 10 " of a random location at $\mathrm{z}=0.225$ is $10^{-4}$. The likely association between GRB050509b and 2MASX J12361286+2858580 is difficult to understand if the GRB resulted from any mechanism involving massive stars and recent star formation, such as a massive-star collapsar or flares by a Soft Gamma Repeater (SGR) magnetar. Infact the galaxy type for the suggested host galaxy is very different than those found for long GRBs; their hosts are typically sub-luminous and blue and show strong emission lines associated with star formation. On the other hand, 2MASX J12361286+2858580 is a very propitious site for a NS-NS or NS-BH merger. In fact, as Chandra observations have shown, giant ellipticals, especially those dominant in their cluster, have very large populations of low mass X-ray binaries (LMXBs) containing accreting neutron stars and black holes. The X-ray afterglow of GRB050509b is the weakest in flux of any of the $15 \mathrm{GRBs}$ that XRT has promptly $(<5 \mathrm{~min})$ observed. If the redshift is 0.225 , then the afterglow is more than 100 times less luminous than that of typical long-burst afterglows. This might be a result of the lower burst energy; the isotropic energy is about $10^{-4}$ of that of typical long GRBs On the other hand, the weak afterglow is consistent with the lower density medium one would expect around an evolved compact binary progenitor. While it is difficult to draw conclusions based on a single object, the Swift localization of GRB050509b near a large elliptical galaxy in a cluster strongly suggests a binary merger origin of at least this one short GRB.

\section{References}

Barthelmy, S. D., 2004, SPIE, 5165

Burrows, D. N., et al., 2004, SPIE, 5165

Burrows, D. N., et al., 2005, Science, 309,1833

Campana, S., et al., 2005, ApJ, 625, L23

Chincarini, G., et al., 2005, ApJ, submitted, astro-ph/0506453

Cusumano, G., et al., 2005, ApJ, submitted

Falcone, A., et al., 2005, ApJ, submitted

Gehrels, N., et al., 2004, ApJ, 611, 1005, astro-ph/0505630

Gehrels, N., et al., 2005, Nature, in press

Moretti, A., et al., 2005, A\&A, submitted

Perri, M., et al., 2005, A\&A, in press, astro-ph/0509164

Nousek, J. A., et al., submitted to ApJ (2005), astro-ph/0508332

Romano, P., et al., 2005, A\&A, submitted

Roming, P. W. A., et al., 2004, SPIE, 5165

Roming, P. W. A., et al., 2005, astro-ph/0509273

Tagliaferri, G., et al., 2005, Nature, 436, 985 


\section{Discussion}

VAN Den Heuvel: Could you tell us a little bit more about the associations between these 3 short bursts with elliptical galaxies, which you mentioned? How good are these associations?

Moretti: Until the end of July we observed 3 short GRB afterglows, 2 detected by Swift (050509b,050724) and 1 detected by HETEII (050709); For 050709 and 050724 the detection of the optical transient and the confirmation of the X-ray afterglow by Chandra observation within a neraby galaxy make the association completely firm (note that the galaxy associated with 050709 is not an elliptical). For 050509b only Swift XRT detected a very faint X-ray afterglow; its position on the sky overlaps a nearby elliptical galaxy. In the error circle (radius 7") other objects are present; however the association of the short GRB with this galaxy seem to be highly probable.

MCBReEn: Do you have redshift measurements for the 3 short GRBs?

MoretTi: The host galaxies of 050509b, 050709, and 050724 are at $\mathrm{z}=0.225, \mathrm{z}=0.16$ and $\mathrm{z}=0.258$, respectively.

Meurs: Just to be certain about these short afterglows: These are all X-ray afterglows but no UVOT detections?

MoretTi: All the afterglow lightcurves I presented are X-ray afterglow light curves as measured by the Swift XRT. 